\title{
A Two-Step Reaction Sequence for the Syntheses of Tetrahydronaphthalenes
}

Gerhard Hilt, * Steffen Lüers and Konstantin I. Smolko

Fachbereich Chemie, Philipps-Universität Marburg, Hans-Meerwein-Straße, 35032

Marburg, Germany

Hilt@chemie.uni-marburg.de

\section{Supporting Information}

\section{General considerations:}

All reactions were carried out with anhydrous solvents in oven dried and argon charged glass ware. Anhydrous dichloromethane was distilled from $\mathrm{P}_{2} \mathrm{O}_{5}$ and toluene from sodium/benzophenone. Solvents used for chromatography and extraction procedures were distilled prior to use.

$\mathrm{CoBr}_{2}$ (dppe) was prepared as reported as reported elsewhere. ${ }^{1}$ Alkynyl pinacolon boronates (2) were prepared using the method of Brown. ${ }^{2}$ Zinc iodide was dried at $160{ }^{\circ} \mathrm{C}$ for $20 \mathrm{~h}$ in high vacuo prior to use. All other chemicals were commercial available and used without further purification, unless indicated.

${ }^{1} \mathrm{H}-\mathrm{NMR}$ spectra were recorded at either $300 \mathrm{MHz}$ on Bruker ARX-300 or at $500 \mathrm{MHz}$ on Bruker ARX-500 spectrometer. ${ }^{13} \mathrm{C}-\mathrm{NMR}$ spectra were recorded at $75 \mathrm{MHz}$ or $126 \mathrm{MHz}$ respectively on the same instruments. NMR spectra were recorded in $\mathrm{CDCl}_{3}$ or actone- $\mathrm{d}_{6}$ with the residue non deuterated solvent peaks taken as internal standard $\left(\delta\left({ }^{1} \mathrm{H}\right)=7.26, \delta\left({ }^{13} \mathrm{C}\right)=\right.$ 77.16 for chloroform and $\delta\left({ }^{1} \mathrm{H}\right)=2.05, \delta\left({ }^{13} \mathrm{C}\right)=29.84$ for acetone), and were recorded in ppm. Abbreviation for signal couplings are as follows: s: singulet, d: doublet, t: triplet, q: quartet, m: multiplet and br for broad signals. Mass spectra were recorded on a Varian $\mathrm{CH} 7$ or a VG Tibrid spectrometer and high resolution mass spectra on a Finnigan-MAT 95S spectrometer. IR spectra were collected on a Bruker IFS 200 or a Nicolet Magna-IR 75 spectrometer as neat films.

Analytical thin layer chromatography was performed on silica gel 60 F254 alumina plates. Column chromatography was performed using silica gel 60 (230-400 mesh).

General procedure for the synthesis of 5-(Hydroxy-(3-nitrophenyl)methyl)-5,8-dimethyl2-phenyl-3a,4,5,9b-tetrahydrobenzo [e]isoindole-1,3-dione (8b, entry 2):
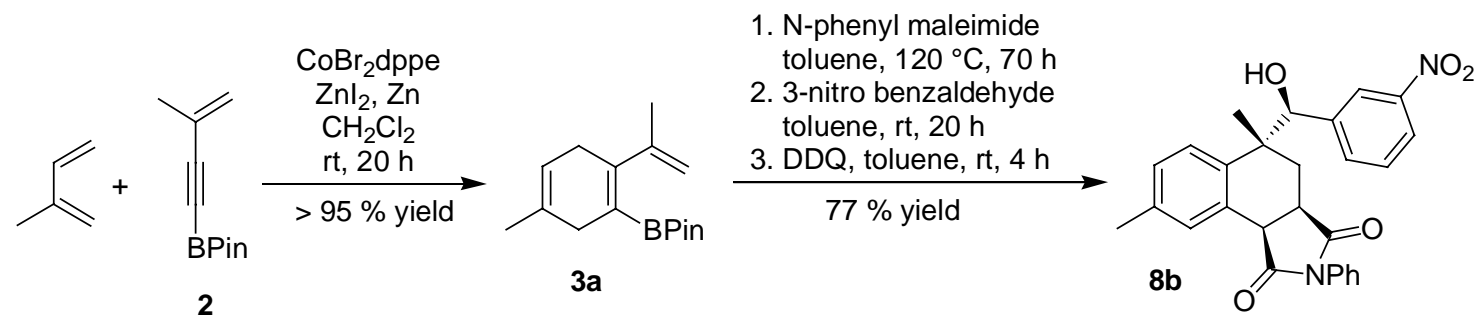
In a Schlenk flask $\mathrm{CoBr}_{2}$ (dppe) $(80 \mathrm{mg}, 0.130 \mathrm{mmol}, 2.5 \mathrm{~mol} \%)$, zinc iodide (200 $\mathrm{mg}, 0.627$ $\mathrm{mmol})$ and zinc dust (50 mg, $0.765 \mathrm{mmol})$ were suspended in dichroromethane $(2.0 \mathrm{ml})$. Then 4,4,5,5-tetramethyl-2-(3-methylbut-3-en-1-ynyl)[1,3,2]dioxaborolane (2) (998 mg, 5.20 mmol) and isoprene (1.00 ml, $680 \mathrm{mg}, 9.97 \mathrm{mmol}, 1.9$ eq.) were added. This mixture was reacted $20 \mathrm{~h}$ at room temperature whereas the color changed from green to deep brown after an induction period of about $10 \mathrm{~min}$. After completion of the reaction diethyl ether was added whereupon the salts precipitated. Filtration through a pad of silica (pentane/diethyl ether $=1 / 1$ ) and removal of the solvents under reduced pressure afforded the air sensitive 1,3-dienyl borolane 3a (> $95 \%$ yield) as an off-white oil in good purity. For further transformations 3a was divided in smaller fractions.

In a sealed tube 1,3-dienyl borolane 3a (188 mg, $0.722 \mathrm{mmol}, 1.0$ eq.) was dissolved in toluene $(0.50 \mathrm{ml})$ and $\mathrm{N}$-phenyl maleimide $(161 \mathrm{mg}, 1.08 \mathrm{mmol}, 1.5 \mathrm{eq}$.$) was added at 0{ }^{\circ} \mathrm{C}$. After warming to room temperature the reaction was heated to $120{ }^{\circ} \mathrm{C}$ for $70 \mathrm{~h}$. Then the mixture was cooled to $0{ }^{\circ} \mathrm{C}$, 3-nitro benzaldehyde $(161 \mathrm{mg}, 1.07 \mathrm{mmol}, 1.5$ eq.) added and reacted at room temperature for $20 \mathrm{~h}$. Afterwards the mixture was diluted with toluene (2.0 $\mathrm{ml})$ and DDQ (310 $\mathrm{mg}, 1.34 \mathrm{mmol}, 1.9$ eq.) added in small portions. The resulting mixture was stirred for $4 \mathrm{~h}$ at room temperature, diluted with dichloromethane and washed with aqueous $\mathrm{NaOH} / \mathrm{Na}_{2} \mathrm{~S}_{2} \mathrm{O}_{3}(10 \% / 10 \%)$ solution. The aqueous layer was twice extracted with dichloromethane and the combined organic phases dried over $\mathrm{MgSO}_{4}$. After removal of the solvents the crude product was prepurified by filtration through a small pad of silica (pentane / ethyl acetate $5 / 1 \rightarrow 1 / 1)$. Recrystallization from methanol yielded the desired tetrahydronaphthalene $\mathbf{8 b}$ ( $254 \mathrm{mg}, 0.55 \mathrm{mmol}, 77 \%)$ as colorless solid.

$\mathbf{R}_{\mathbf{f}}($ pentane $/$ ethyl acetate $=1 / 1)=0.67$.

${ }^{1}$ H-NMR $\left(300 \mathrm{MHz}, \mathrm{CDCl}_{3}\right): \delta=8.04(\mathrm{~d}, J=7.3 \mathrm{~Hz}, 1 \mathrm{H}), 7.81(\mathrm{~s}, 1 \mathrm{H}), 7.70(\mathrm{~s}, 1 \mathrm{H}), 7.42$ $7.25(\mathrm{~m}, 5 \mathrm{H}), 7.22-7.15(\mathrm{~m}, 2 \mathrm{H}), 6.90(\mathrm{~d}, J=8.0 \mathrm{~Hz}, 1 \mathrm{H}), 6.67(\mathrm{~d}, J=8.0 \mathrm{~Hz}, 1 \mathrm{H}), 4.82(\mathrm{~s}$, $1 \mathrm{H}), 4.15$ (d, $J=6.8 \mathrm{~Hz}, 1 \mathrm{H}), 3.67$ (br.s, $1 \mathrm{H}), 2.58$ (dd, $J=13.5,5.0 \mathrm{~Hz}, 1 \mathrm{H}$ ), 2.43 (br.s, 1 $\mathrm{H}), 2.30(\mathrm{~s}, 3 \mathrm{H}), 1.53(\mathrm{t}, J=12.3 \mathrm{~Hz}, 1 \mathrm{H}), 1.13(\mathrm{~s}, 3 \mathrm{H})$.

${ }^{13}$ C-NMR $\left(75 \mathrm{MHz}, \mathrm{CDCl}_{3}\right): \delta=178.6(\mathrm{C}=\mathrm{O}), 176.0(\mathrm{C}=\mathrm{O}), 147.6\left(\mathrm{C}_{\mathrm{q}}\right), 142.4\left(\mathrm{C}_{\mathrm{q}}\right), 137.3$ $\left(\mathrm{C}_{\mathrm{q}}\right), 137.2\left(\mathrm{C}_{\mathrm{q}}\right), 133.9(\mathrm{CH}), 132.0\left(\mathrm{C}_{\mathrm{q}}\right), 130.9(\mathrm{CH}), 129.2(2 \mathrm{CH}), 129.0\left(\mathrm{C}_{\mathrm{q}}\right), 128.7(\mathrm{CH})$, $128.5(\mathrm{CH}), 128.4(\mathrm{CH}), 126.5(2 \mathrm{CH}), 126.3(\mathrm{CH}), 122.9(\mathrm{CH}), 122.7(\mathrm{CH}), 78.2(\mathrm{OCH})$, $42.3(\mathrm{CH}), 41.7\left(\mathrm{C}_{\mathrm{q}}\right), 37.6(\mathrm{CH}), 33.4\left(\mathrm{CH}_{2}\right), 22.4\left(\mathrm{CH}_{3}\right), 21.1\left(\mathrm{CH}_{3}\right)$.

MS (ESI): $\mathrm{m} / \mathrm{z}(\%)=479\left(100,[\mathrm{M}+\mathrm{Na}]^{+}\right)$.

HRMS (ESI): calcd for $\mathrm{C}_{27} \mathrm{H}_{24} \mathrm{~N}_{2} \mathrm{O}_{5} \mathrm{Na}: 479.1583 \mathrm{~m} / \mathrm{z}$ [M+Na] ${ }^{+}$; found: $479.1578 \mathrm{~m} / \mathrm{z}$.

IR (neat): 3479 (s), 2973 (w), 1778 (m), 1710 (s), 1597 (w), 1528 (s), 1498 (s), 1383 (s), 1350 (s), $908(\mathrm{w}), 845(\mathrm{w}), 814(\mathrm{w}), 731(\mathrm{~s}), 694(\mathrm{~s})$.

\section{5-(Hydroxy-(2-nitrophenyl)methyl)-5,8-dimethyl-2-phenyl-3a,4,5,9b-tetrahydrobenzo [e]isoindole-1,3-dione (8a, entry 1):}

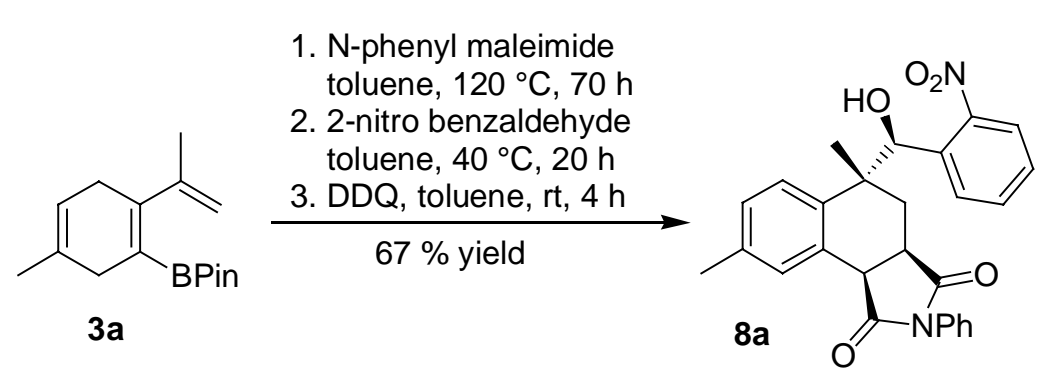

Following the general procedure 1,3-dienyl borolane 3a (198 mg, $0.761 \mathrm{mmol}, 1.0$ eq.), Nphenyl maleimide (161 mg, $1.01 \mathrm{mmol}, 1.3$ eq.), 2-nitro benzaldehyde (148 mg, $0.979 \mathrm{mmol}$, 
1.3 eq.) and DDQ (181 mg, $0.783 \mathrm{mmol}, 1.0$ eq.) were brought to reaction. The allylation was carried out at $40^{\circ} \mathrm{C}$. Recrystallization from chloroform yielded the desired tetrahydronaphthalene 8b (233 mg, $0.510 \mathrm{mmol}, 67 \%$ ) as light yellow solid.

$\mathbf{R}_{\mathbf{f}}($ pentane / ethyl acetate $=1 / 1)=0.66$.

${ }^{1}$ H-NMR $\left(300 \mathrm{MHz}, \mathrm{CDCl}_{3}\right): \delta=7.90(\mathrm{dd}, J=8.0,1.2 \mathrm{~Hz}, 1 \mathrm{H}), 7.72(\mathrm{~s}, 1 \mathrm{H}), 7.61(\mathrm{td}, J=$ 7.5, $1.2 \mathrm{~Hz}, 1 \mathrm{H}), 7.55(\mathrm{dd}, J=8.3,1.2 \mathrm{~Hz}, 1 \mathrm{H}), 7.43-7.28(\mathrm{~m}, 4 \mathrm{H}), 7.23-7.18(\mathrm{~m}, 2 \mathrm{H}), 6.75$ $(\mathrm{d}, J=8.1 \mathrm{~Hz}, 1 \mathrm{H}), 6.29(\mathrm{~d}, J=8.1 \mathrm{~Hz}, 1 \mathrm{H}), 6.02(\mathrm{~s}, 1 \mathrm{H}), 4.14(\mathrm{~d}, J=8.8 \mathrm{~Hz}, 1 \mathrm{H}), 3.69$ (ddd, $J=13.4,8.8,5.9 \mathrm{~Hz}, 1 \mathrm{H}), 2.78$ (dd, $J=13.4,5.9 \mathrm{~Hz}, 1 \mathrm{H}), 2.28$ (s, $3 \mathrm{H}$ ), 1.60 (br.s, 1 $\mathrm{H}), 1.50(\mathrm{t}, J=13.4 \mathrm{~Hz}, 1 \mathrm{H}), 1.16(\mathrm{~s}, 3 \mathrm{H})$.

${ }^{13}$ C-NMR $\left(75 \mathrm{MHz}, \mathrm{CDCl}_{3}\right): \delta=178.7(\mathrm{C}=\mathrm{O}), 176.1(\mathrm{C}=\mathrm{O}), 148.9\left(\mathrm{C}_{\mathrm{q}}\right), 137.3\left(\mathrm{C}_{\mathrm{q}}\right), 136.4$ $\left(\mathrm{C}_{\mathrm{q}}\right), 135.7\left(\mathrm{C}_{\mathrm{q}}\right), 132.4(\mathrm{CH}), 132.1\left(\mathrm{C}_{\mathrm{q}}\right), 130.6(\mathrm{CH}), 129.8(\mathrm{CH}), 129.5\left(\mathrm{C}_{\mathrm{q}}\right), 129.2(2 \mathrm{CH})$, $128.7(\mathrm{CH}), 128.4(\mathrm{CH}), 128.2(\mathrm{CH}), 126.6(2 \mathrm{CH}), 126.4(\mathrm{CH}), 124.1(\mathrm{CH}), 70.0(\mathrm{OCH})$, $42.3\left(\mathrm{C}_{\mathrm{q}}\right), 41.9(\mathrm{CH}), 37.6(\mathrm{CH}), 35.1\left(\mathrm{CH}_{2}\right), 21.2\left(\mathrm{CH}_{3}\right), 20.9\left(\mathrm{CH}_{3}\right)$.

MS (ESI): $\mathrm{m} / \mathrm{z}(\%)=455(73), 479\left(23,[\mathrm{M}+\mathrm{Na}]^{+}\right), 568(100)$.

HRMS (ESI): calcd for $\mathrm{C}_{27} \mathrm{H}_{24} \mathrm{~N}_{2} \mathrm{O}_{5} \mathrm{Na}: 479.1583 \mathrm{~m} / \mathrm{z}$ [M+Na] ${ }^{+}$; found: $479.1591 \mathrm{~m} / \mathrm{z}$.

IR (neat): 3545 (m), 2957 (w), 1773 (m), 1704 (ss), 1595 (w), 1577 (w), 1529 (s), 1497 (s), 1385 (s), 1347 (s), 1187 (s), 1027 (s), 823 (w), 792 (m), 748 (m), 702 (m).

\section{5-(Hydroxy-(4-nitrophenyl)methyl)-5,8-dimethyl-2-phenyl-3a,4,5,9b-tetrahydrobenzo[e] isoindole-1,3-dione (8c, entry 3):}

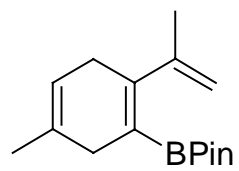

$3 a$

\section{N-phenyl maleimide} toluene, $120^{\circ} \mathrm{C}, 70 \mathrm{~h}$

2. 4-nitro benzaldehyde toluene, $40^{\circ} \mathrm{C}, 20 \mathrm{~h}$

3. DDQ, toluene, $\mathrm{rt}, 4 \mathrm{~h}$ $48 \%$ yield

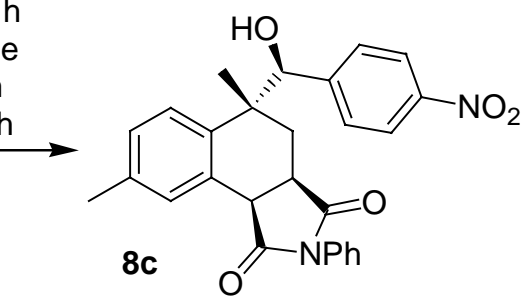

Following the general procedure 1,3-dienyl borolane 3a (212 mg, $0.815 \mathrm{mmol}, 1.0$ eq.), Nphenyl maleimide (171 mg, $0.987 \mathrm{mmol}, 1.2$ eq.), 4-nitro benzaldehyde (180 mg, $1.19 \mathrm{mmol}$, 1.5 eq.) and DDQ (196 mg, $0.848 \mathrm{mmol}, 1.0$ eq.) were brought to reaction. The allylation was carried out at $40{ }^{\circ} \mathrm{C}$. Recrystallization from methanol yielded the desired tetrahydronaphthalene 8c (180 mg, $0.394 \mathrm{mmol}, 48 \%$ ) as colorless solid.

$\mathbf{R}_{\mathbf{f}}($ pentane / ethyl acetate $=1 / 1)=0.71$

${ }^{1}$ H-NMR (300 MHz, acetone-d $\left.\mathrm{d}_{6}\right): \delta=8.08(\mathrm{~d}, J=9.0 \mathrm{~Hz}, 2 \mathrm{H}), 7.80(\mathrm{~s}, 1 \mathrm{H}), 7.52-7.31$ (m, 5 H), $7.25(\mathrm{~d}, J=9.0 \mathrm{~Hz}, 2 \mathrm{H}), 6.89(\mathrm{~d}, J=8.0 \mathrm{~Hz}, 1 \mathrm{H}), 6.64(\mathrm{~d}, J=8.0 \mathrm{~Hz}, 1 \mathrm{H}), 5.09-5.02$ $(\mathrm{m}, 2 \mathrm{H}), 4.52(\mathrm{~d}, J=9.0 \mathrm{~Hz}, 1 \mathrm{H}), 3.88(\mathrm{ddd}, J=13.3,9.0,6.0 \mathrm{~Hz}, 1 \mathrm{H}), 2.75(\mathrm{dd}, J=13.3$, $6.0 \mathrm{~Hz}, 1 \mathrm{H}), 2.33(\mathrm{~s}, 3 \mathrm{H}), 1.66(\mathrm{t}, J=13.3 \mathrm{~Hz}, 1 \mathrm{H}), 1.26$ (s, $3 \mathrm{H})$.

${ }^{13}$ C-NMR $\left(75 \mathrm{MHz}\right.$, acetone- $\left.\mathrm{d}_{6}\right): \delta=179.1(\mathrm{C}=\mathrm{O}), 176.9(\mathrm{C}=\mathrm{O}), 150.7\left(\mathrm{C}_{\mathrm{q}}\right), 148.1\left(\mathrm{C}_{\mathrm{q}}\right)$, $139.1\left(\mathrm{C}_{\mathrm{q}}\right), 136.7\left(\mathrm{C}_{\mathrm{q}}\right), 134.1\left(\mathrm{C}_{\mathrm{q}}\right), 131.3(\mathrm{CH}), 131.0\left(\mathrm{C}_{\mathrm{q}}\right), 130.1(2 \mathrm{CH}), 129.6(2 \mathrm{CH}), 129.0$ $(\mathrm{CH}), 128.1(\mathrm{CH}), 128.0(2 \mathrm{CH}), 127.7(\mathrm{CH}), 122.8(2 \mathrm{CH}), 77.7(\mathrm{OCH}), 43.0(\mathrm{CH}), 42.7$ $\left(\mathrm{C}_{\mathrm{q}}\right), 38.4(\mathrm{CH}), 35.2\left(\mathrm{CH}_{2}\right), 22.3\left(\mathrm{CH}_{3}\right), 21.5\left(\mathrm{CH}_{3}\right)$.

MS (ESI): $\mathrm{m} / \mathrm{z}(\%)=372(100), 479\left(14,[\mathrm{M}+\mathrm{Na}]^{+}\right)$.

HRMS (ESI): calcd for $\mathrm{C}_{27} \mathrm{H}_{24} \mathrm{~N}_{2} \mathrm{O}_{5} \mathrm{Na}: 479.1583 \mathrm{~m} / \mathrm{z}$ [M+Na] ${ }^{+}$; found: $479.1598 \mathrm{~m} / \mathrm{z}$.

IR (neat): 3542 (m), 2921 (w), 1774 (w), 1708 (s), 1599 (w), 1517 (s), 1498 (m), 1385 (s), 1346 (s), 1192 (s), 863 (w), 846 (w), 800 (w), 747 (m), 700 (m). 
5-(4-Chlorophenyl)hydroxy methyl-5,8-dimethyl-2-phenyl-3a,4,5,9b-tetrahydrobenzo[e] isoindole-1,3-dione (8d, entry 4):

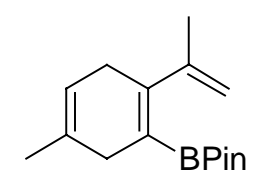

$3 a$

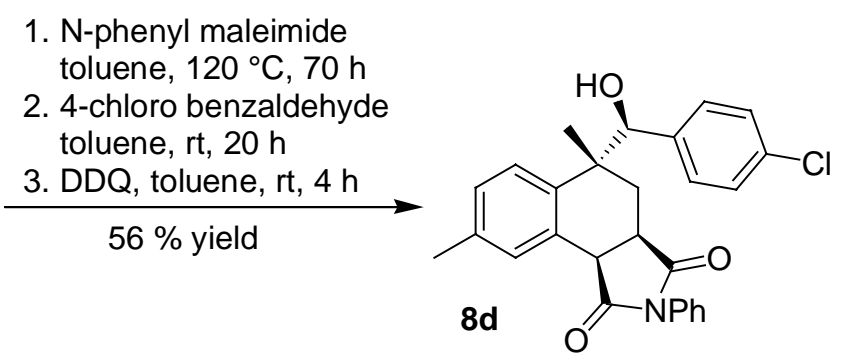

Following the general procedure 1,3-dienyl borolane 3a (232 mg, $0.892 \mathrm{mmol}, 1.0$ eq.), Nphenyl maleimide (175 mg, $1.01 \mathrm{mmol}, 1.1 \mathrm{eq}$.), 4-chloro benzaldehyde (160 mg, $1.14 \mathrm{mmol}$, $1.3 \mathrm{eq})$ and DDQ (350 mg, $1.51 \mathrm{mmol}, 1.7 \mathrm{eq}$.) were brought to reaction. Recrystallization from chloroform yielded the desired tetrahydronaphthalene $8 d$ (224 mg, $0.502 \mathrm{mmol}, 56 \%)$ as colorless solid.

$\mathbf{R}_{\mathbf{f}}$ (pentane / ethyl acetate $\left.=1 / 1\right)=0.71$.

${ }^{1} \mathbf{H}-\mathbf{N M R}\left(500 \mathrm{MHz}, \mathrm{CDCl}_{3}\right): \delta=7.69(\mathrm{~s}, 1 \mathrm{H}), 7.38(\mathrm{t}, J=7.7 \mathrm{~Hz}, 2 \mathrm{H}), 7.30(\mathrm{t}, J=7.4 \mathrm{~Hz}, 1$ H), 7.23-7.18 (m, $4 \mathrm{H}), 7.22-6.96(\mathrm{~m}, 3 \mathrm{H}), 6.89(\mathrm{~d}, J=8.0 \mathrm{~Hz}, 1 \mathrm{H}), 4.72(\mathrm{~s}, 1 \mathrm{H}), 4.12(\mathrm{~d}, J$ $=8.9 \mathrm{~Hz}, 1 \mathrm{H}), 3.67(\mathrm{ddd}, J=12.0,8.9,6.2 \mathrm{~Hz}, 1 \mathrm{H}), 2.54(\mathrm{dd}, J=13.7,6.2 \mathrm{~Hz}, 1 \mathrm{H}), 2.32(\mathrm{~s}$, $3 \mathrm{H}), 1.85$ (br.s, $1 \mathrm{H}), 1.50$ (dd, $J=13.7,12.0 \mathrm{~Hz} 1 \mathrm{H}), 1.14$ (s, $3 \mathrm{H})$.

${ }^{13}$ C-NMR $\left(126 \mathrm{MHz}, \mathrm{CDCl}_{3}\right): \delta=178.5(\mathrm{C}=\mathrm{O}), 176.1(\mathrm{C}=\mathrm{O}), 138.7\left(\mathrm{C}_{\mathrm{q}}\right), 137.9\left(\mathrm{C}_{\mathrm{q}}\right), 137.0$ $\left(\mathrm{C}_{\mathrm{q}}\right), 133.8\left(\mathrm{C}_{\mathrm{q}}\right), 132.1\left(\mathrm{C}_{\mathrm{q}}\right), 130.9(\mathrm{CH}), 129.3\left(\mathrm{C}_{\mathrm{q}}\right), 129.3(2 \mathrm{CH}), 129.2(2 \mathrm{CH}), 128.7(\mathrm{CH})$, $128.6(\mathrm{CH}), 128.1(2 \mathrm{CH}), 126.5(2 \mathrm{CH}), 126.4(\mathrm{CH}), 79.8(\mathrm{OCH}), 42.6(\mathrm{CH}), 41.6\left(\mathrm{C}_{\mathrm{q}}\right), 38.0$ $(\mathrm{CH}), 33.2\left(\mathrm{CH}_{2}\right), 23.9\left(\mathrm{CH}_{3}\right), 21.2\left(\mathrm{CH}_{3}\right)$.

MS (ESI): $\mathrm{m} / \mathrm{z}(\%)=163(100), 468\left(21,[\mathrm{M}+\mathrm{Na}]^{+}\right)$.

HRMS (ESI): calcd for $\mathrm{C}_{27} \mathrm{H}_{24} \mathrm{ClNO}_{3} \mathrm{Na}: 468.1342 \mathrm{~m} / \mathrm{z}[\mathrm{M}+\mathrm{Na}]^{+}$; found: $468.1362 \mathrm{~m} / \mathrm{z}$.

IR (neat): 3548 (s), 1775 (m), 1708 (ss), 1491 (s), 1387 (s), 1192 (s), 829 (m), 797 (m), 747 (m), $702(\mathrm{~m})$.

\section{5-(Hydroxy-(3-nitrophenyl)methyl)-5,7,8-trimethyl-2-phenyl-3a,4,5,9b-tetrahydrobenzo} [e]isoindole-1,3-dione (8e, entry 5):

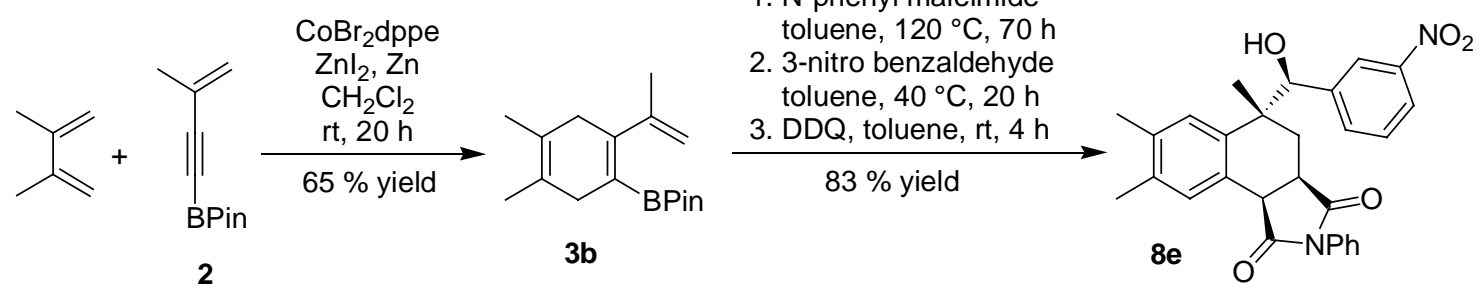

The 1,3-dienyl borolane $\mathbf{3 b}$ was prepared on a $5.00 \mathrm{mmol}$ scale analogous to $\mathbf{3 a}$ using 2,3dimethyl butadiene instead of isoprene. Column chromatography (pentane / tert-butyl methyl ether: 100:1) afforded 3b in $65 \%$ yield as colorless oil. For further transformations $\mathbf{3 b}$ was divided in smaller fractions.

Following the general procedure 1,3-dienyl borolane $\mathbf{3 b}$ ( $155 \mathrm{mg}, 0.565 \mathrm{mmol}, 1.0$ eq.), Nphenyl maleimide (117 mg, $0.676 \mathrm{mmol}, 1.2 \mathrm{eq}$.), 3-nitro benzaldehyde (125 mg, 0.827 mmol, 1.5 eq.) and DDQ (280 mg, $1.22 \mathrm{mmol}, 2.1$ eq.) were brought to reaction. The allylation was carried out at $40{ }^{\circ} \mathrm{C}$. Column chromatography $\left(\mathrm{SiO}_{2}\right.$, pentane / ethyl acetate $5 / 1 \rightarrow 1 / 1)$ yielded the desired tetrahydronaphthalene $8 \mathbf{e}(220 \mathrm{mg}, 0.468 \mathrm{mmol}$, $83 \%)$ as colorless solid. 
$\mathbf{R}_{\mathbf{f}}($ pentane / ethyl acetate $=1 / 1)=0.67$.

${ }^{1}$ H-NMR $\left(300 \mathrm{MHz}, \mathrm{CDCl}_{3}\right): \delta=8.04(\mathrm{dt}, J=7.6,1.7 \mathrm{~Hz}, 1 \mathrm{H}), 7.77(\mathrm{~s}, 1 \mathrm{H}), 7.62(\mathrm{~s}, 1 \mathrm{H})$, 7.41-7.25 (m, $5 \mathrm{H}), 7.21-7.13(\mathrm{~m}, 2 \mathrm{H}), 6.53(\mathrm{~s}, 1 \mathrm{H}), 4.81(\mathrm{~s}, 1 \mathrm{H}), 4.11(\mathrm{~d}, J=8.8 \mathrm{~Hz}, 1 \mathrm{H})$, 3.64 (ddd, $J=11.7,8.8,6.4 \mathrm{~Hz}, 1 \mathrm{H}), 2.53$ (dd, $J=13.7,6.4 \mathrm{~Hz}, 1 \mathrm{H}), 2.42$ (br.s, $1 \mathrm{H}$ ), 2.21 $(\mathrm{s}, 3 \mathrm{H}), 2.03(\mathrm{~s}, 3 \mathrm{H}), 1.51(\mathrm{dd}, J=13.7,11.7 \mathrm{~Hz}, 1 \mathrm{H}), 1.10(\mathrm{~s}, 3 \mathrm{H})$.

${ }^{13}$ C-NMR $\left(75 \mathrm{MHz}, \mathrm{CDCl}_{3}\right): \delta=178.6(\mathrm{C}=\mathrm{O}), 176.1(\mathrm{C}=\mathrm{O}), 147.6\left(\mathrm{C}_{\mathrm{q}}\right), 142.4\left(\mathrm{C}_{\mathrm{q}}\right), 137.5$ $\left(\mathrm{C}_{\mathrm{q}}\right), 136.2\left(\mathrm{C}_{\mathrm{q}}\right), 136.1\left(\mathrm{C}_{\mathrm{q}}\right), 133.9(\mathrm{CH}), 132.1\left(\mathrm{C}_{\mathrm{q}}\right), 131.5\left(\mathrm{C}_{\mathrm{q}}\right), 129.2(2 \mathrm{CH}), 128.7(\mathrm{CH})$, $128.4(\mathrm{CH}), 127.4(\mathrm{CH}), 126.5(\mathrm{CH}), 126.4(2 \mathrm{CH}), 123.0(\mathrm{CH}), 122.7(\mathrm{CH}), 78.4(\mathrm{OCH})$, $42.1(\mathrm{CH}), 41.7\left(\mathrm{C}_{\mathrm{q}}\right), 37.7(\mathrm{CH}), 33.1\left(\mathrm{CH}_{2}\right), 22.8\left(\mathrm{CH}_{3}\right), 19.6\left(\mathrm{CH}_{3}\right), 19.5\left(\mathrm{CH}_{3}\right)$.

MS (ESI): $\mathrm{m} / \mathrm{z}(\%)=493\left(100,[\mathrm{M}+\mathrm{Na}]^{+}\right)$.

HRMS (ESI): calcd for $\mathrm{C}_{28} \mathrm{H}_{26} \mathrm{~N}_{2} \mathrm{O}_{5} \mathrm{Na}: 493.1739 \mathrm{~m} / \mathrm{z}$ [M+Na] ${ }^{+}$; found: $493.1736 \mathrm{~m} / \mathrm{z}$.

IR (neat): 3495 (m), 3380 (m), 2917 (m), 1775 (m), 1714 (s), 1598 (w), 1524 (s), 1499 (s), 1455 (m), 1388 (s), 1348 (s), 906 (w), 842 (w), 799 (w), 745 (s), 727 (s), 691 (s).

5-(1-Hydroxypropyl)-5,8-dimethyl-2-phenyl-3a,4,5,9b-tetrahydrobenzo[e]isoindole-1,3dione (8f, entry 6):
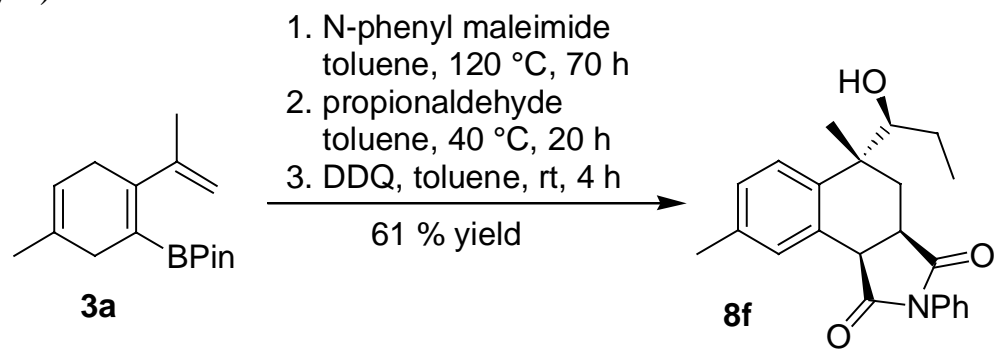

Following the general procedure 1,3-dienyl borolane 3a (205 mg, $0.788 \mathrm{mmol}, 1.0$ eq.), Nphenyl maleimide (177 mg, $1.02 \mathrm{mmol}, 1.3 \mathrm{eq}$.$) , propionaldehyde (0.30 \mathrm{ml}, 242 \mathrm{mg}, 4.16$ mmol, 5.3 eq.) and DDQ (240 mg, $1.04 \mathrm{mmol}, 1.3$ eq.) were brought to reaction. The allylation was carried out at $40{ }^{\circ} \mathrm{C}$. Recrystallization from methanol / water yielded the desired tetrahydronaphthalene $\mathbf{8 f}$ (175 $\mathrm{mg}, 0.483 \mathrm{mmol}, 61 \%)$ as colorless solid.

mp $164-166^{\circ} \mathrm{C}$.

$\mathbf{R}_{\mathbf{f}}($ pentane $/$ ethyl acetate $=1 / 1)=0.80$.

${ }^{1} \mathbf{H}-\mathbf{N M R}\left(300 \mathrm{MHz}, \mathrm{CDCl}_{3}\right): \delta=7.67(\mathrm{~s}, 1 \mathrm{H}), 7.42-7.27(\mathrm{~m}, 3 \mathrm{H}), 7.23-7.16(\mathrm{~m}, 3 \mathrm{H}), 7.06$ $(\mathrm{d}, J=8.1 \mathrm{~Hz}, 1 \mathrm{H}), 4.06(\mathrm{~d}, J=8.8 \mathrm{~Hz}, 1 \mathrm{H}), 3.65(\mathrm{ddd}, J=11.5,8.8,6.1 \mathrm{~Hz}, 1 \mathrm{H}), 3.43(\mathrm{dd}$, $J=10.3,2.0 \mathrm{~Hz}, 1 \mathrm{H}), 2.48(\mathrm{dd}, J=13.7,6.1 \mathrm{~Hz}, 1 \mathrm{H}), 2.30(\mathrm{~s}, 3 \mathrm{H}), 1.63-1.41(\mathrm{~m}, 4 \mathrm{H}), 1.20$ $(\mathrm{s}, 3 \mathrm{H}), 0.94(\mathrm{t}, J=7.2 \mathrm{~Hz}, 3 \mathrm{H})$.

${ }^{13}$ C-NMR $\left(75 \mathrm{MHz}, \mathrm{CDCl}_{3}\right): \delta=178.9(\mathrm{C}=\mathrm{O}), 176.3(\mathrm{C}=\mathrm{O}), 139.3\left(\mathrm{C}_{\mathrm{q}}\right), 136.6\left(\mathrm{C}_{\mathrm{q}}\right), 132.2$ $\left(\mathrm{C}_{\mathrm{q}}\right), 130.9(\mathrm{CH}), 129.6\left(\mathrm{C}_{\mathrm{q}}\right), 129.2(2 \mathrm{CH}), 128.7(\mathrm{CH}), 128.6(\mathrm{CH}), 126.5(2 \mathrm{CH}), 125.6$ $(\mathrm{CH}), 80.0(\mathrm{OCH}), 42.6(\mathrm{CH}), 41.2\left(\mathrm{C}_{\mathrm{q}}\right), 37.8(\mathrm{CH}), 33.0\left(\mathrm{CH}_{2}\right), 24.0\left(\mathrm{CH}_{3}\right), 23.9\left(\mathrm{CH}_{2}\right), 21.2$ $\left(\mathrm{CH}_{3}\right), 11.8\left(\mathrm{CH}_{3}\right)$.

MS (ESI): $\mathrm{m} / \mathrm{z}(\%)=386\left(43,[\mathrm{M}+\mathrm{Na}]^{+}\right), 479(100)$.

HRMS (ESI): calcd for $\mathrm{C}_{23} \mathrm{H}_{25} \mathrm{NO}_{3} \mathrm{Na}: 386.1732 \mathrm{~m} / \mathrm{z}$ [M+Na] ${ }^{+}$; found: $386.1740 \mathrm{~m} / \mathrm{z}$.

IR (neat): 3529 (s), 2972 (m), 1778 (w), 1708 (s), 1597 (w), 1496 (m), 1381 (s), 750 (m). 


\section{5-(1-Hydroxyallyl)-5,8-dimethyl-2-phenyl-3a,4,5,9b-tetrahydrobenzo[e]isoindole-1,3-}

dione $(8 g$, entry 7$)$ :

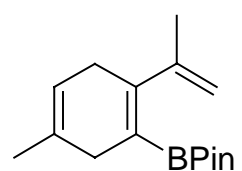

$3 a$
1. N-phenyl maleimide toluene, $120^{\circ} \mathrm{C}, 70 \mathrm{~h}$

2. acroleine toluene, $40^{\circ} \mathrm{C}, 20 \mathrm{~h}$

3. DDQ, toluene, rt, $4 \mathrm{~h}$
$71 \%$ yield

Following the general procedure 1,3-dienyl borolane 3a (205 mg, $0.788 \mathrm{mmol}, 1.0$ eq.), Nphenyl maleimide $(163 \mathrm{mg}, 0.941 \mathrm{mmol}, 1.2 \mathrm{eq}$.$) , freshly destilled acroleine (0.30 \mathrm{ml}, 252$ $\mathrm{mg}, 4.50 \mathrm{mmol}, 5.7$ eq.) and DDQ (260 mg, $1.13 \mathrm{mmol}, 1.4$ eq.) were brought to reaction. The allylation was carried out at $40{ }^{\circ} \mathrm{C}$. Recrystallization from tert-butyl methyl ether yielded the desired tetrahydronaphthalene $\mathbf{8 g}$ (202 $\mathrm{mg}, 0.559 \mathrm{mmol}, 71 \%)$ as colorless solid.

mp $177-179^{\circ} \mathrm{C}$.

$\mathbf{R}_{\mathbf{f}}($ pentane $/$ ethyl acetate $=1 / 1)=0.82$.

${ }^{1}$ H-NMR (300 MHz, CDCl 3 ): $\delta=7.66(\mathrm{~s}, 1 \mathrm{H}), 7.41-7.25(\mathrm{~m}, 3 \mathrm{H}), 7.22-7.16(\mathrm{~m}, 3 \mathrm{H}), 7.05$ $(\mathrm{d}, J=8.1 \mathrm{~Hz}, 1 \mathrm{H}), 5.91$ (ddd, $J=16.8,10.5,5.9 \mathrm{~Hz}, 1 \mathrm{H}), 5.30-5.18(\mathrm{~m}, 2 \mathrm{H}), 4.10(\mathrm{~d}, J=$ $5.9 \mathrm{~Hz}, 1 \mathrm{H}), 4.06(\mathrm{~d}, J=8.8 \mathrm{~Hz}, 1 \mathrm{H}), 3.65(\mathrm{ddd}, J=11.6,8.8,6.3 \mathrm{~Hz}, 1 \mathrm{H}), 2.46(\mathrm{dd}, J=$ 13.7, $6.3 \mathrm{~Hz}, 1 \mathrm{H}), 2.29$ (s, $3 \mathrm{H}), 1.65$ (s, $1 \mathrm{H}), 1.55$ (dd, $J=13.7,11.6 \mathrm{~Hz}, 1 \mathrm{H}), 1.18$ (s, $3 \mathrm{H})$.

${ }^{13}$ C-NMR $\left(75 \mathrm{MHz}, \mathrm{CDCl}_{3}\right): \delta=178.7(\mathrm{C}=\mathrm{O}), 176.2(\mathrm{C}=\mathrm{O}), 138.5\left(\mathrm{C}_{\mathrm{q}}\right), 136.7\left(\mathrm{C}_{\mathrm{q}}\right), 136.3$ $(\mathrm{CH}), 132.2\left(\mathrm{C}_{\mathrm{q}}\right), 130.9(\mathrm{CH}), 129.6\left(\mathrm{C}_{\mathrm{q}}\right), 129.2(2 \mathrm{CH}), 128.7(\mathrm{CH}), 128.6(\mathrm{CH}), 126.5(2$ $\mathrm{CH}), 125.8(\mathrm{CH}), 117.5\left(\mathrm{CH}_{2}\right), 79.2(\mathrm{OCH}), 42.5(\mathrm{CH}), 40.8\left(\mathrm{C}_{\mathrm{q}}\right), 37.9(\mathrm{CH}), 33.0\left(\mathrm{CH}_{2}\right)$, $24.2\left(\mathrm{CH}_{3}\right), 21.2\left(\mathrm{CH}_{3}\right)$.

MS (ESI): $\mathrm{m} / \mathrm{z}(\%)=363\left(25,[\mathrm{M}+2 \mathrm{H}]^{+}\right), 384\left(100,[\mathrm{M}+\mathrm{Na}]^{+}\right)$.

HRMS (ESI): calcd for $\mathrm{C}_{23} \mathrm{H}_{23} \mathrm{NO}_{3} \mathrm{Na}: 384.1576 \mathrm{~m} / \mathrm{z}[\mathrm{M}+\mathrm{Na}]^{+}$; found: $384.1575 \mathrm{~m} / \mathrm{z}$.

IR (neat): 3410 (s), 3074 (w), 2973 (w), 2884 (w), 1770 (w), 1698 (ss), 1598 (w), 1498 (s), 1389 (s), 1197 (s), 1004 (w), 918 (m), 786 (w), 743 (m), 690 (w).

\section{5-Hydroxymethyl-5,8-dimethyl-2-phenyl-3a,4,5,9b-tetrahydrobenzo[e]isoindole-1,3-} dione (8h, entry 8):

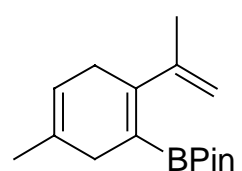

3a

\section{N-phenyl maleimide} toluene, $120^{\circ} \mathrm{C}, 70 \mathrm{~h}$

2. $p$-formaldehyde toluene, $100^{\circ} \mathrm{C}, 20 \mathrm{~h}$ 3. DDQ, toluene, rt, $4 \mathrm{~h}$

$69 \%$ yield

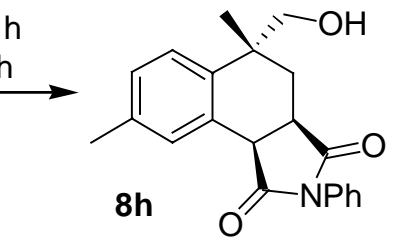

Following the general procedure 1,3-dienyl borolane 3a (215 mg, $0.826 \mathrm{mmol}, 1.0$ eq.), Nphenyl maleimide (198 mg, $1.14 \mathrm{mmol}, 1.4$ eq.), $p$-formaldehyde (161 mg) and DDQ (240 $\mathrm{mg}, 1.07 \mathrm{mmol}, 1.3 \mathrm{eq}$.) were brought to reaction. The allylation was carried out at $100{ }^{\circ} \mathrm{C}$. Recrystallization from methanol yielded the desired tetrahydronaphthalene $\mathbf{8 h}$ (191 mg, 0.569 mmol, $69 \%$ ) as colorless solid.

mp $82-83{ }^{\circ} \mathrm{C}$.

$\mathbf{R}_{\mathbf{f}}($ pentane $/$ ethyl acetate $=1 / 1)=0.52$. 
${ }^{1}$ H-NMR (300 MHz, $\left.\mathrm{CDCl}_{3}\right): \delta=7.56(\mathrm{~s}, 1 \mathrm{H}), 7.41-7.25(\mathrm{~m}, 3 \mathrm{H}), 7.22-7.13(\mathrm{~m}, 3 \mathrm{H}), 7.04$ $(\mathrm{d}, J=8.1 \mathrm{~Hz}, 1 \mathrm{H}), 4.02(\mathrm{~d}, J=8.7 \mathrm{~Hz}, 1 \mathrm{H}), 3.57(\mathrm{~d}, J=11.2 \mathrm{~Hz}, 1 \mathrm{H}), 3.52-3.39(\mathrm{~m}, 2 \mathrm{H})$, $2.35(\mathrm{dd}, J=13.7,6.4 \mathrm{~Hz}, 1 \mathrm{H}), 2.28$ (s, $3 \mathrm{H}), 1.97$ (s, $1 \mathrm{H}), 1.69$ (dd, $J=13.7,10.4 \mathrm{~Hz}, 1 \mathrm{H})$, 1.19 (s, $3 \mathrm{H})$.

${ }^{13}$ C-NMR $\left(75 \mathrm{MHz}, \mathrm{CDCl}_{3}\right): \delta=178.9(\mathrm{C}=\mathrm{O}), 176.1(\mathrm{C}=\mathrm{O}), 138.0\left(\mathrm{C}_{\mathrm{q}}\right), 136.7\left(\mathrm{C}_{\mathrm{q}}\right), 132.1$ $\left(\mathrm{C}_{\mathrm{q}}\right), 131.2(\mathrm{CH}), 129.2(2 \mathrm{CH}), 128.9\left(\mathrm{C}_{\mathrm{q}}\right), 128.8(\mathrm{CH}), 128.6(\mathrm{CH}), 126.4(2 \mathrm{CH}), 125.5$ $(\mathrm{CH}), 70.4\left(\mathrm{OCH}_{2}\right), 42.7(\mathrm{CH}), 38.7\left(\mathrm{C}_{\mathrm{q}}\right), 37.8(\mathrm{CH}), 34.0\left(\mathrm{CH}_{2}\right), 24.9\left(\mathrm{CH}_{3}\right), 21.1\left(\mathrm{CH}_{3}\right)$.

MS (ESI): $\mathrm{m} / \mathrm{z}(\%)=358\left(100,[\mathrm{M}+\mathrm{Na}]^{+}\right)$.

HRMS (ESI): calcd for $\mathrm{C}_{21} \mathrm{H}_{21} \mathrm{NO}_{3} \mathrm{Na}: 358.1419 \mathrm{~m} / \mathrm{z}[\mathrm{M}+\mathrm{Na}]^{+}$; found: $358.1418 \mathrm{~m} / \mathrm{z}$.

IR (neat): 3469 (m), 2923 (m), 1776 (w), 1711 (s), 1597 (w), 1499 (s), 1382 (s), 1181 (s), 909 (w), $847(\mathrm{w}), 818(\mathrm{w}), 745(\mathrm{~m}), 691(\mathrm{~m})$.

4-(Hydroxy-(3-nitrophenyl)methyl)-4-methyl-1,2,3,4-tetrahydronaphthalene-1,2-dicarbo nitrile (81, entry 12$)$ :

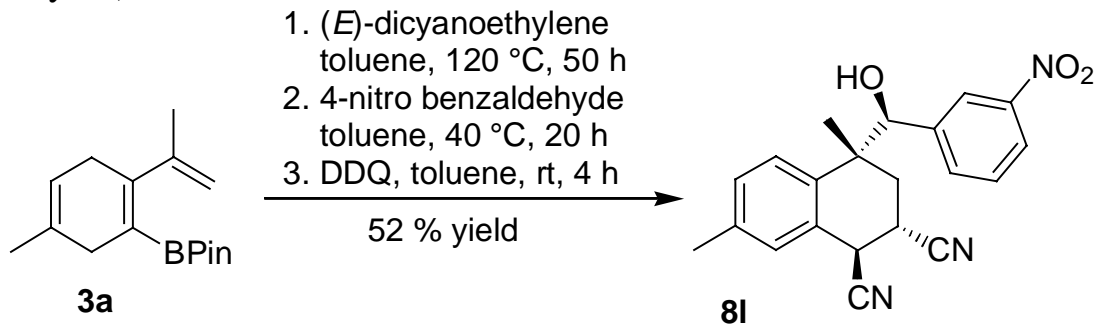

Following the general procedure 1,3-dienyl borolane 3a (69 mg, $0.265 \mathrm{mmol}, 1.0$ eq.) and (E)-dicyanoethylene $(25 \mathrm{mg}, 0.320 \mathrm{mmol}, 1.2 \mathrm{eq}$.) were reacted in toluene $(0.20 \mathrm{ml})$ for $50 \mathrm{~h}$ at $120{ }^{\circ} \mathrm{C}$. The allylation with 3-nitro benzaldehyde $(50 \mathrm{mg}, 0.404 \mathrm{mmol}, 1.5 \mathrm{eq})$ was carried out at $40{ }^{\circ} \mathrm{C}$. After oxidation with DDQ $(90 \mathrm{mg}, 0.397 \mathrm{mmol}, 1.5$ eq.) the reaction was worked up with $\mathrm{NaOH} / \mathrm{Na}_{2} \mathrm{~S}_{2} \mathrm{O}_{3}(0.1 \mathrm{M} / 0.1 \mathrm{M})$. Recrystallization from chloroform yielded the desired tetrahydronaphthalene $\mathbf{8 m}(51 \mathrm{mg}, 0.138 \mathrm{mmol}, 52 \%)$ as light brown solid.

$\mathbf{R}_{\mathbf{f}}($ pentane $/$ ethyl acetate $=1 / 1)=0.48$.

${ }^{1}$ H-NMR (300 MHz, acetone-d $\left.\mathrm{d}_{6}\right): \delta=8.36(\mathrm{t}, J=2.0 \mathrm{~Hz}, 1 \mathrm{H}), 8.19$ (ddd, $J=8.1,2.2,1.0 \mathrm{~Hz}$, $1 \mathrm{H}), 7.96(\mathrm{~d}, J=7.8 \mathrm{~Hz}, 1 \mathrm{H}), 7.75(\mathrm{~d}, J=8.1 \mathrm{~Hz}, 1 \mathrm{H}), 7.67(\mathrm{t}, J=7.9 \mathrm{~Hz}, 1 \mathrm{H}), 7.31(\mathrm{~s}, 1$ H), $7.26(\mathrm{~d}, J=8.1 \mathrm{~Hz}, 1 \mathrm{H}), 5.47(\mathrm{~d}, 1 \mathrm{H}, J=4.1 \mathrm{~Hz}, 1 \mathrm{H}), 4.78(\mathrm{~d}, J=4.1 \mathrm{~Hz}, 1 \mathrm{H}), 4.61(\mathrm{~d}$, $J=11.9 \mathrm{~Hz}, 1 \mathrm{H}), 3.53(\mathrm{ddd}, J=13.2,11.9,2.7 \mathrm{~Hz}, 1 \mathrm{H}), 2.70(\mathrm{t}, J=13.2 \mathrm{~Hz}, 1 \mathrm{H}), 2.37(\mathrm{~s}, 3$ $\mathrm{H}), 1.65(\mathrm{dd}, J=13.4,2.7 \mathrm{~Hz}, 1 \mathrm{H}), 1.34(\mathrm{~s}, 3 \mathrm{H})$.

${ }^{13}$ C-NMR $\left(75 \mathrm{MHz}\right.$, acetone-d $\left.\mathrm{d}_{6}\right): \delta=148.8\left(\mathrm{C}_{\mathrm{q}}\right), 144.8\left(\mathrm{C}_{\mathrm{q}}\right), 139.0\left(\mathrm{C}_{\mathrm{q}}\right), 137.3\left(\mathrm{C}_{\mathrm{q}}\right), 135.4$ $(\mathrm{CH}), 130.6(\mathrm{CH}), 129.7(\mathrm{CH}), 129.5\left(\mathrm{C}_{\mathrm{q}}\right), 128.7(\mathrm{CH}), 128.5(\mathrm{CH}), 123.6(\mathrm{CH}), 123.2(\mathrm{CH})$, $120.7(\mathrm{CN}), 119.9(\mathrm{CN}), 78.9(\mathrm{OCH}), 43.9\left(\mathrm{C}_{\mathrm{q}}\right), 36.8(\mathrm{CH}), 33.5\left(\mathrm{CH}_{2}\right), 28.4\left(\mathrm{CH}_{3}\right), 28.1$ $(\mathrm{CH}), 20.9\left(\mathrm{CH}_{3}\right)$.

MS (ESI): $\mathrm{m} / \mathrm{z}(\%)=384\left(100,[\mathrm{M}+\mathrm{Na}]^{+}\right), 400\left(54,[\mathrm{M}+\mathrm{K}]^{+}\right)$.

This material isomerizes partially upon recrystallization from methanol. 


\section{5-(Hydroxy(phenyl)methyl)-5,8-dimethyl-3a,4,5,6,9,9b-hexahydronaphtho[1,2-c]furan-}

1,3-dione (7i, entry 9):

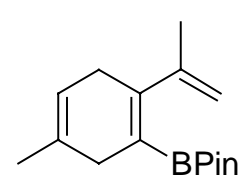

3a
1. maleic anhydrid toluene, $100^{\circ} \mathrm{C}, 120 \mathrm{~h}$

2. benzaldehyde

toluene, $40^{\circ} \mathrm{C}, 2 \mathrm{~h}$

Following the general procedure 1,3-dienyl borolane 3a (200 mg, $0.769 \mathrm{mmol}, 1.0 \mathrm{eq}$.$) and$ maleic anhydrid $(75 \mathrm{mg}, 0.765 \mathrm{mmol}, 1.0$ eq.) were reacted in toluene $(12 \mathrm{ml})$ for $120 \mathrm{~h}$ at $100{ }^{\circ} \mathrm{C}$. The allylation with benzaldehyde $(0.50 \mathrm{ml}, 522 \mathrm{mg}, 4.91 \mathrm{mmol}, 6.4 \mathrm{eq})$ was carried out at $40{ }^{\circ} \mathrm{C}$ for $2 \mathrm{~h}$. After removing of the solvent the crude product was dried under reduced pressure for $12 \mathrm{~h}$. Column chromatography $\left(\mathrm{SiO}_{2}\right.$, pentane / tert-butyl methy ether $\left.7 / 3\right)$ yielded the desired hexahydronaphthalene $7 \mathbf{i}(154 \mathrm{mg}, 0.455 \mathrm{mmol}, 59 \%)$ as colorless solid.

${ }^{1}$ H-NMR (500 MHz, acetone- $\left.\mathrm{d}_{6}\right): \delta=7.39(\mathrm{~d}, J=7.2 \mathrm{~Hz}, 2 \mathrm{H}), 7.32(\mathrm{t}, J=7.4 \mathrm{~Hz}, 2 \mathrm{H}), 7.26$ (tt, $J=7.3,1.6 \mathrm{~Hz}, 1 \mathrm{H}), 5.45-5.41(\mathrm{~m}, 1 \mathrm{H}), 4.89(\mathrm{~s}, 1 \mathrm{H}), 4.12$ (br.s, $1 \mathrm{H}), 3.67$ (ddd, $J=$ $13.4,5.6,3.1 \mathrm{~Hz}, 1 \mathrm{H}), 3.28(\mathrm{~d}, J=5.5 \mathrm{~Hz}, 1 \mathrm{H}), 2.96-2.74(\mathrm{~m}, 3 \mathrm{H}), 2.59(\mathrm{dt}, J=20.8,6.7$ $\mathrm{Hz}, 1 \mathrm{H}), 2.14(\mathrm{dd}, J=14.2,2.1 \mathrm{~Hz}, 1 \mathrm{H}), 1.76(\mathrm{t}, J=13.7 \mathrm{~Hz}, 1 \mathrm{H}), 1.68$ (s, $3 \mathrm{H}), 0.91$ (s, 3 $\mathrm{H})$.

${ }^{13} \mathrm{C}-\mathrm{NMR}\left(75 \mathrm{MHz}\right.$, acetone- $\left.\mathrm{d}_{6}\right): \delta=174.6(\mathrm{C}=\mathrm{O}), 173.9(\mathrm{C}=\mathrm{O}), 143.3\left(\mathrm{C}_{\mathrm{q}}\right), 133.8\left(\mathrm{C}_{\mathrm{q}}\right), 131.0$ $\left(\mathrm{C}_{\mathrm{q}}\right), 129.0(2 \mathrm{CH}), 128.1(2 \mathrm{CH}), 127.8(\mathrm{CH}), 126.4\left(\mathrm{C}_{\mathrm{q}}\right), 119.3(\mathrm{CH}), 79.2(\mathrm{OCH}), 51.5$ $(\mathrm{CH}), 48.1(\mathrm{CH}), 43.0\left(\mathrm{C}_{\mathrm{q}}\right), 39.6\left(\mathrm{CH}_{2}\right), 37.2\left(\mathrm{CH}_{2}\right), 27.0\left(\mathrm{CH}_{2}\right), 25.6\left(\mathrm{CH}_{3}\right), 22.7\left(\mathrm{CH}_{3}\right)$.

\section{4-((4-Chlorophenyl) hydroxy methyl)-4,7-dimethyl-1,2,3,4-tetrahydro naphthalene-1,2-} dicarboxylic acid dimethyl ester (8m, entry 13$)$ :

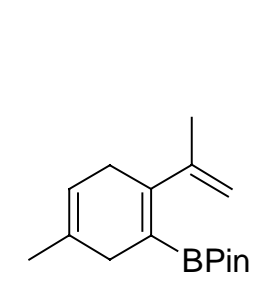

$3 a$

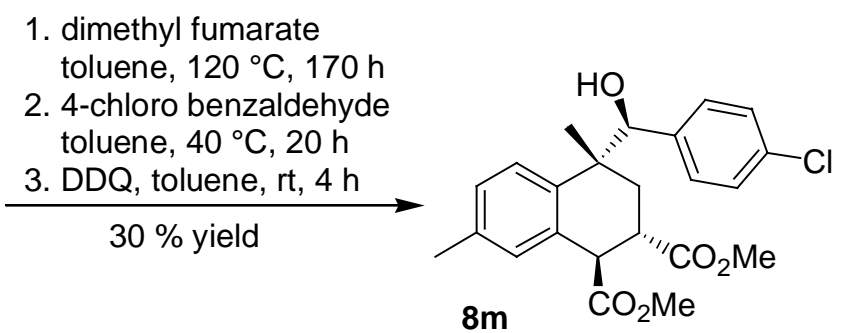

$8 m$

Following the general procedure 1,3-dienyl borolane 3a (212 mg, $0.815 \mathrm{mmol}, 1.0$ eq.) and dimethyl fumarate $(150 \mathrm{mg}, 1.04 \mathrm{mmol}, 1.3 \mathrm{eq}$.) were reacted in toluene $(1.0 \mathrm{ml})$ for $170 \mathrm{~h}$ at $120{ }^{\circ} \mathrm{C}$. The allylation with 4-chloro benzaldehyde $(180 \mathrm{mg}, 1.28 \mathrm{mmol}, 1.6 \mathrm{eq})$ was carried out at $40{ }^{\circ} \mathrm{C}$. After oxidation with DDQ (268 mg, $1.18 \mathrm{mmol}, 1.5$ eq.) the reaction was worked up as usual. Column chromatography $\left(\mathrm{SiO}_{2}\right.$, pentane / ethyl acetate $\left.20 / 1 \rightarrow 1 / 1\right)$ yielded a diastereomeric product mixture (ratio $\sim 1: 1$ ) and a small quantity of dihydroaromatic by-product (102 $\mathrm{mg}, 0.245 \mathrm{mmol}, 30 \%$ ) as colorless solid.

${ }^{1} \mathrm{H}-\mathrm{NMR}\left(300 \mathrm{MHZ}, \mathrm{CDCl}_{3}\right)$ : signals of the product mixture: $\delta=7.36(\mathrm{~d}, 1 \mathrm{H}), 7.28-6.85(\mathrm{~m}$, $6 \mathrm{H})$, 5.41-5.36 (m, $1 \mathrm{H}), 5.34-5.28(\mathrm{~m}, 1 \mathrm{H}), 5.06(\mathrm{~s}, 1 \mathrm{H}), 4.86(\mathrm{~s}, 1 \mathrm{H}), 4.83(\mathrm{~s}, 1 \mathrm{H}), 4.79$ $(\mathrm{s}, 1 \mathrm{H}), 4.20-0.90(\mathrm{~m}, 8 \mathrm{H}), 4.13(\mathrm{~d}, 1 \mathrm{H}), 3.96(\mathrm{~d}, 1 \mathrm{H}), 3.73(\mathrm{~s}, 3 \mathrm{H}), 3.71(\mathrm{~s}, 3 \mathrm{H}), 3.66(\mathrm{~s}, 3$ H), $3.64(\mathrm{~s}, 3 \mathrm{H}), 3.61(\mathrm{~s}, 3 \mathrm{H}), 3.57(\mathrm{~s}, 3 \mathrm{H}), 3.52(\mathrm{~s}, 3 \mathrm{H}), 3.28(\mathrm{~d}, 1 \mathrm{H}), 2.23(\mathrm{~s}, 3 \mathrm{H}), 1.84$ (s, $3 \mathrm{H}), 1.82$ (s, $3 \mathrm{H}), 1.18$ (s, $3 \mathrm{H}), 1.11$ (s, $3 \mathrm{H}), 1.07$ (s, $3 \mathrm{H}), 0.97$ (s, $3 \mathrm{H})$. 
X-ray structure of 8d (entry 4):

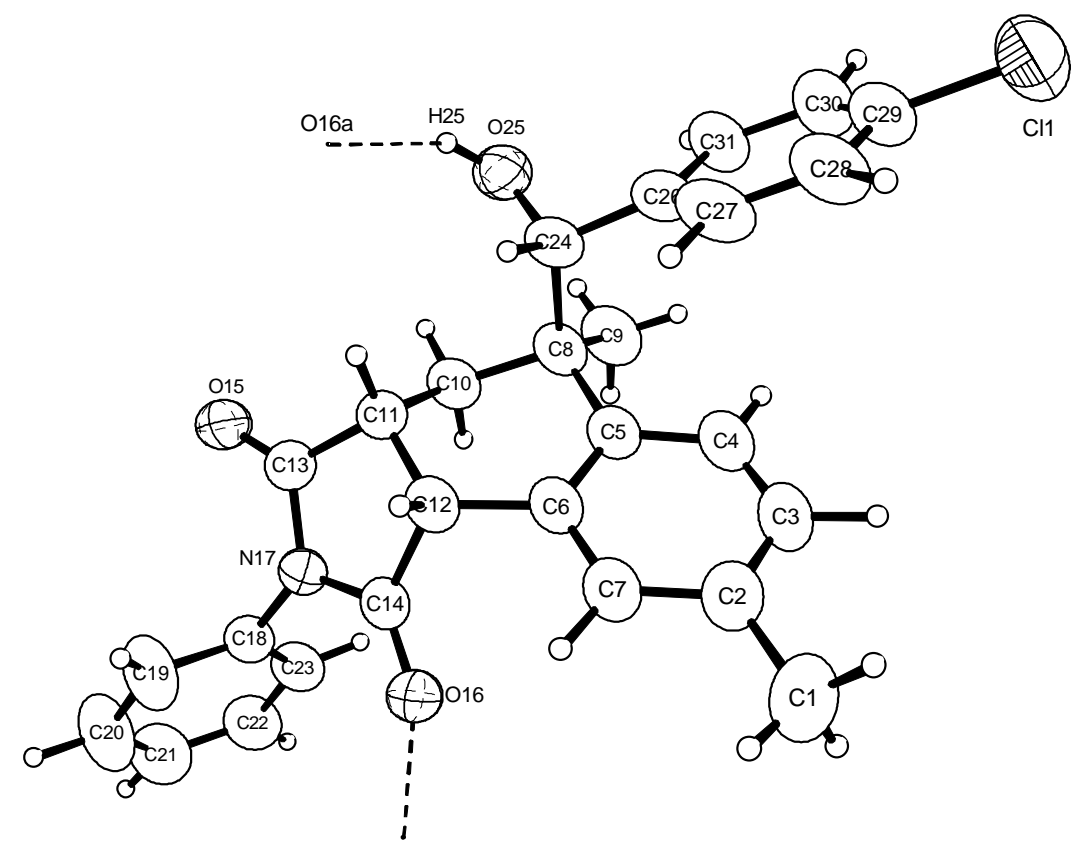

${ }^{1}$ G. Hilt, S. Lüers, Synthesis 2002, 609.

${ }^{2}$ H. C. Brown, N. G. Bhat, M. Srebnik, Tetrahedron Lett. 1988, 29, 2631. 